\title{
Sine-Gordon/Coulomb Gas Soliton Correlation Functions and an Exact Evaluation of the Kosterlitz-Thouless Critical Exponent
}

\author{
Leonardo Mondaini* and E. C. Marino ${ }^{\dagger}$ \\ Instituto de Física, Universidade Federal do Rio de Janeiro \\ Cx.Postal 68528, Rio de Janeiro RJ 21941-972, Brazil
}

\begin{abstract}
We present an exact derivation for the asymptotic large distance behavior of the spin two-point correlation function in the XY-model. This allows for the exact obtainment of the critical exponent $\eta=$ $1 / 4$ at the Kosterlitz-Thouless transition that occurs in this model and in the 2D neutral Coulomb gas and which has been previously obtained by scaling arguments. In order to do that, we use the language of sine-Gordon theory to obtain a Coulomb Gas description of the XY-model spin correlation function, which becomes identified with the soliton correlator of that theory. Using a representation in terms of bipolar coordinates we obtain an exact expression for the asymptotic large distance behavior of the relevant correlator at $\beta^{2}=8 \pi$, which corresponds to the Kosterlitz-Thouless transition. The result is obtained by approaching this point from the plasma (high-temperature) phase of the gas. The vortex correlator of the $\mathrm{XY}$-model is also obtained using the same procedure.
\end{abstract}

KEY WORDS: soliton correlation functions, sine-Gordon theory, XY-model, 2D Coulomb gas, Kosterlitz-Thouless transition

\section{INTRODUCTION}

The sine-Gordon (SG) model is certainly one of the best studied of $(1+1)$ dimensional physics. The interest in this field theoretical model has been enhanced by its connections with the two-dimensional (2D) neutral Coulomb gas (CG) 1] and also with the 2D XY-magnetic system [2]. In this framework, it

\footnotetext{
*mondaini@if.ufrj.br

†marino@if.ufrj.br
} 
becomes an useful and powerful tool for the study of a great variety of physical properties of these two systems, which in principle admit actual realizations in nature. The SG model is integrable in the sense that the spectrum and the Smatrix are exactly known 3. Nevertheless, there is a lack of exact results for correlation functions, except for a specific value of the coupling parameter 4 .

Many interesting results have been obtained recently, concerning the SG system. The thermodynamic Bethe ansatz has been used for obtaining the free energy and specific heat of the system 5 . Exact form factors of the soliton operators and other fields have been derived 6, 7]. Density correlation functions have been calculated using these form factors 8 . Several results concerning the thermodynamics of the classical CG have also been obtained 9]. Among these, we mention the exact free energy for $\beta^{2}<4 \pi$, in the case of point particles 10 and for $4 \pi<\beta^{2}<6 \pi$, in the case of extensive ones 11. Charge and particle correlators have been obtained in the low temperature $\left(\beta^{2}>8 \pi\right)$ phase [12.

In this work we present a computation of the two-point spin correlation function of the XY-model using its connection to the SG theory. In particular, we exploit the relation existing between the spin operators of the former and the soliton creation operators of the latter. Then, we use the CG representation of these functions in order to derive an exact series describing the large distance behavior of them. This series clearly exhibits two distinct types of asymptotic behavior, separated by a critical point at $\beta^{2}=8 \pi$. At this point, we obtain an exact result for the spin-spin correlator of the XY-model, which exhibits a power-law behavior with exponent $\eta=\frac{1}{4}$, well-known from the scaling analysis of the XY-model 2]. This special point, indeed, is the Kosterlitz-Thouless (KT) critical point [13] and, in the 2D CG language, it corresponds to the temperature $T_{c}$ in which the system undergoes a phase transition from a metallic (or plasma) phase, composed of charged particles, into an insulating (or dieletric) phase, composed of neutral dipoles (bound pairs of charges). For $\beta^{2}<8 \pi$ (hightemperature), our series exhibits a nontrivial large distance behavior, whereas for $\beta^{2}>8 \pi$ (low-temperature), its large distance behavior is determined by the free theory, as expected.

The KT critical exponent is usually obtained through scaling arguments, which are approximate, in the low-temperature phase [2 14 15] 16] or by heuristic arguments in the high-temperature phase [17. The result presented here is the first exact evaluation of the KT critical exponent, performed by approaching the critical point from the high-temperature (in CG language) phase.

In section 2 , we review the connection between the SG theory with the $2 \mathrm{D}$ $\mathrm{CG}$ and also with the magnetic XY-model. We pay special attention to the correspondence between the soliton creation operators in the SG theory and the spin operators in the 2D XY-model. This allows us to establish the equivalence between their respective correlation functions, which can be written in a convenient form with the help of the 2D CG picture. In section 3, we make use of bipolar coordinates in order to put the correlation functions in a form that easily allows for the obtainment of an exact asymptotic solution at the KT critical point. In section 4 , we consider the case of the vortex correlation function. Finally, some concluding remarks are presented in section 5. Two appendices are included. In Appendix A, we review the details of the system of bipolar coordinates em- 
ployed in the calculations, whereas in Appendix B, we demonstrate properties of the functions $K(|\vec{x}-\vec{y}|)$ and $K^{\prime}(|\vec{x}-\vec{y}|)$ appearing in our final expressions for the soliton and the vortex correlation functions, respectively, which are used in obtaining the asymptotic behavior of these correlators.

\section{SOLITON AND SPIN OPERATORS}

In this section, we are going to review the connection of the SG theory with the 2D neutral CG and with the 2D XY-model. We will highlight, in particular, the relation of the soliton creation operator of that theory with the spin operator of the latter model, as well as the representation of its correlation functions in the framework of the classical CG. This will be our starting point for the evaluation of correlation functions at the KT point.

We start from the SG euclidean action, given by

$$
S=\int d x d \tau\left[\frac{1}{2} \partial_{\mu} \phi \partial_{\mu} \phi+2 \alpha_{0} \cos \beta \phi\right] .
$$

It is well-known that the vacuum functional of the theory may be written as 11. 15.

$$
\mathcal{Z}=\lim _{\varepsilon \rightarrow 0} \sum_{n=0}^{\infty} \frac{\alpha^{2 n}}{(n !)^{2}} \int \prod_{i=1}^{2 n} d^{2} z_{i} \exp \left\{\frac{\beta^{2}}{8 \pi} \sum_{i \neq j=1}^{2 n} \lambda_{i} \lambda_{j} \ln \left[\left|\vec{z}_{i}-\vec{z}_{j}\right|^{2}+\varepsilon^{2}\right]\right\},
$$

where $\lambda_{i}=1$ for $1 \leq i \leq n$ and $\lambda_{i}=-1$ for $n+1 \leq i \leq 2 n$ and $\varepsilon$ is a shortdistance regulator, which is needed in the case of point particles or, equivalently, of a local field theory. The renormalized coupling $\alpha$ is related to the one in (1) by

$$
\alpha=\alpha_{0}\left(\varepsilon^{2}\right)^{\frac{\beta^{2}}{8 \pi}} .
$$

In expression (2) we recognize $\mathcal{Z}$ as the grand-partition function for the classical 2D CG of point particles with charges \pm 1 , where $\alpha$ is the fugacity and $\frac{\beta^{2}}{2 \pi}=\frac{1}{k T_{C G}}$, where $T_{C G}$ is the Coulomb gas temperature. In obtaining this relation, we are using the convention of 18 for defining the $2 \mathrm{D}$ Coulomb potential. In the case of particles with a finite diameter, $d$, the integration region for the $2 n z_{i}$-integrals in (2) must exclude the regions where $\left|\vec{z}_{i}-\vec{z}_{j}\right|<d$.

The theory possesses soliton excitations, bearing a topological charge

$$
Q=\frac{\beta}{2 \pi} \int d x \partial_{x} \phi
$$

At the quantum level, the corresponding states are created by the soliton operator of Mandelstam [19]

$$
\mu(x, \tau)=\exp \left\{i \frac{2 \pi}{\beta} \int_{-\infty}^{x} d z \dot{\phi}(z, \tau)\right\} .
$$

As we will see below, this operator has an interesting physical interpretation in terms of the associated spin system, namely, the XY-model. 
The exact soliton mass spectrum is known, as well as the masses of soliton bound states (breathers), which occur for $\beta^{2}<4 \pi[3$. Their masses are given by

$$
M_{n}=2 M \sin \left(\frac{\pi \xi}{2} n\right) \quad, \quad n=1,2, \ldots<\frac{1}{\xi}
$$

where $\xi=\frac{\beta^{2}}{8 \pi-\beta^{2}}$ and $M$ is the soliton mass.

The SG/CG system is closely related to a $2 \mathrm{D}$ spin system, namely the $\mathrm{O}(2)$ ferromagnetic Heisenberg model on a square lattice, or XY-model [2]. This is described by the hamiltonian

$$
H_{X Y}=-J \sum_{\langle i j\rangle} \vec{n}_{i} \cdot \vec{n}_{j}
$$

where [2, 20]

$$
\vec{n}_{i}=\left(\cos \sqrt{\frac{T}{2 J}} \theta_{i}, \sin \sqrt{\frac{T}{2 J}} \theta_{i}\right) .
$$

The sum in (7) runs over nearest neighbors and $T$ is the temperature (note that the temperature in the XY-model, $T$, is not the same as in the $\left.\mathrm{CG}, T_{C G}\right)$. Observe that $\left|\vec{n}_{i}\right|^{2}=1$ and $\theta_{i}$ is proportional to the angle the spin $\vec{n}_{i}$ makes with the $\mathrm{x}$-axis.

When we take the continuum limit of (7) and consider the contributions of both spin-waves and vortices to the total energy, we arrive at the hamiltonian 2

$$
H_{X Y}=\int d^{2} x\left[\frac{1}{2} \vec{\nabla} \theta \cdot \vec{\nabla} \theta+\kappa \cos \left(2 \pi \sqrt{\frac{2 J}{T}} \phi\right)\right],
$$

where we have considered that $\theta_{i} \rightarrow \theta(\vec{x})$ in the continuum limit and $\phi$ is a scalar field related to $\theta(\vec{x})$ by

$$
\theta(x, y)=\int_{-\infty}^{x} d z \partial_{y} \phi(z, y)
$$

From (10), it follows that

$$
\vec{\nabla} \theta \cdot \vec{\nabla} \theta=\vec{\nabla} \phi \cdot \vec{\nabla} \phi
$$

and we see, by comparing (9) with (1), that the hamiltonian of the XY-model coincides with the euclidean action of the SG model, provided we make the identifications: $\beta=2 \pi \sqrt{\frac{2 J}{T}}$ and $\kappa=2 \alpha_{0}$. It also follows that the euclidean vacuum functional of the SG theory, the grand-partition function of the $2 \mathrm{D}$ CG and the partition function of the XY-model are all identified.

In the XY-model, similarly to the case of the CG of extensive particles, we have a natural short distance cutoff, namely, the lattice spacing $\tau$ [2]. Accordingly, the integration region for the $2 n z_{i}$-integrals in (2) must exclude, in this case, the regions where $\left|\vec{z}_{i}-\vec{z}_{j}\right|<\tau$.

Within this picture, a very interesting and useful connection can be established between the XY-spin thermal correlation functions and the SG quantum 
soliton correlators. Indeed, in view of (10) we see that the SG soliton operator (5) can be written as

$$
\mu(x, \tau)=\exp \left\{i \frac{2 \pi}{\beta} \theta(x, \tau)\right\} .
$$

The relevant XY-correlator, therefore, may be written as

$$
\langle\vec{n}(\vec{r}) \cdot \vec{n}(\overrightarrow{0})\rangle_{X Y}=\left\langle\mu(\vec{r}) \mu^{\dagger}(\overrightarrow{0})\right\rangle_{S G},
$$

where the first expression is the XY-thermal correlator, whereas the second one is the SG quantum soliton correlator.

Within the CG description, the soliton correlator (13) is given by [15]:

$$
\begin{array}{r}
\left\langle\mu(\vec{x}) \mu^{\dagger}(\vec{y})\right\rangle=\frac{\mathcal{Z}^{-1}}{|\vec{x}-\vec{y}|^{\frac{2 \pi}{\beta^{2}}}} \sum_{n=0}^{\infty} \frac{\alpha^{2 n}}{(n !)^{2}} \int_{V(r)} \prod_{i=1}^{2 n} d^{2} z_{i} \\
\times \exp \left\{\frac{\beta^{2}}{8 \pi} \sum_{i \neq j=1}^{2 n} \lambda_{i} \lambda_{j} \ln \left|\vec{z}_{i}-\vec{z}_{j}\right|^{2}+i \sum_{i=1}^{2 n} \lambda_{i}\left[\arg \left(\vec{z}_{i}-\vec{y}\right)-\arg \left(\vec{z}_{i}-\vec{x}\right)\right]\right\},
\end{array}
$$

where $\mathcal{Z}^{-1}$ is given by (2). In the previous expression, we are considering either the case of the XY-model or the CG of extensive particles, where, as we have mentioned, there is a natural short distance cutoff. Consequently, the integrals in (14) and in $\mathcal{Z}$ are defined in the region $V(r)$, in which $r<\left|\vec{z}_{i}-\vec{z}_{j}\right|<R, r$ being the lattice spacing $\tau$ in the former case and the particle diameter $d$ in the latter, whereas $R$ is the radius of the system. $V(r)$, therefore defines a finite "volume" $\left(V \simeq \pi R^{2}\right.$ since $r<<R$ ) for the system. We have droped the regulator $\varepsilon$ from the Coulomb potential used in (14) and in $\mathcal{Z}$, since it is no longer needed in the presence of the natural short distance cutoff $r$.

Observe that, in (14) the contribution coming from the soliton operators at $\vec{x}$ and $\vec{y}$ corresponds, in the CG language, to the interaction of the charges of the gas with an external string of electric dipoles orthogonal to it, going from $\vec{x}$ to $\vec{y}$, plus the self-interaction of this string, which consists in the first term in (14) [21].

\section{THE SOLITON CORRELATION FUNCTION}

In this section, we are going to obtain a representation of the soliton correlator (14), valid for $\vec{x} \neq \vec{y}$, which will enable us to derive an exact expression for the asymptotic large distance behavior of $\left\langle\mu \mu^{\dagger}\right\rangle$ at the KT point, $\beta^{2}=8 \pi$.

Indeed, for $\vec{x} \neq \vec{y}$ we may use the bipolar coordinates described in the Appendix A, and rewrite (14) as

$$
\begin{aligned}
& \left\langle\mu(\vec{x}) \mu^{\dagger}(\vec{y})\right\rangle=\frac{\mathcal{Z}^{-1}}{|\vec{x}-\vec{y}|^{\frac{2 \pi}{\beta^{2}}}} \sum_{n=0}^{\infty} \frac{\alpha^{2 n}}{(n !)^{2}} \int_{0, V(r)}^{2 \pi} \int_{-\infty, V(r)}^{+\infty} \prod_{i=1}^{2 n} d \xi_{i} d \eta_{i} \frac{|\vec{x}-\vec{y}|^{4 n}}{4\left[\cosh \eta_{i}-\cos \xi_{i}\right]^{2}} \\
& \times \exp \left\{\frac { \beta ^ { 2 } } { 8 \pi } \sum _ { i \neq j = 1 } ^ { 2 n } \lambda _ { i } \lambda _ { j } \operatorname { l n } \left\{| \vec { x } - \vec { y } | ^ { 2 } \left[\left(\frac{\sinh \eta_{i}}{2\left[\cosh \eta_{i}-\cos \xi_{i}\right]}-\frac{\sinh \eta_{j}}{2\left[\cosh \eta_{j}-\cos \xi_{j}\right]}\right)^{2}\right.\right.\right.
\end{aligned}
$$




$$
\left.\left.\left.+\left(\frac{\sin \xi_{i}}{2\left[\cosh \eta_{i}-\cos \xi_{i}\right]}-\frac{\sin \xi_{j}}{2\left[\cosh \eta_{j}-\cos \xi_{j}\right]}\right)^{2}\right]\right\}+i \sum_{i=1}^{2 n} \lambda_{i} \xi_{i}\right\} .
$$

In this expression, as before, the symbol $V(r)$ expresses the fact that the integrations must respect the condition that $r<\left|\vec{z}_{i}-\vec{z}_{j}\right|<R$. In terms of the $\xi_{i}, \eta_{i}$ integrals, this implies the following restriction for the expressions between round brackets in 15, which we call, respectively $\alpha_{i j}$ and $\beta_{i j}$ :

$$
\left[\alpha_{i j}^{2}+\beta_{i j}^{2}\right] \in\left[\frac{r^{2}}{|\vec{x}-\vec{y}|^{2}}, \frac{R^{2}}{|\vec{x}-\vec{y}|^{2}}\right] \stackrel{|\vec{x}-\vec{y}|>>r}{\longrightarrow}\left[\frac{r^{2}}{4 R^{2}}, \frac{1}{4}\right] .
$$

By inspecting (15), we immediately conclude that the $|\vec{x}-\vec{y}|$-factors decouple from the $\xi_{i}, \eta_{i}$ integrals. Elementary combinatorics, taking into account the neutrality of the gas, shows that this factor will appear $n(n-1)$ times in the numerator and $n^{2}$ times in the denominator. Combining with the $4 n$ contribution coming from the scale factors of the volume elements, we obtain

$$
\left\langle\mu(\vec{x}) \mu^{\dagger}(\vec{y})\right\rangle=\frac{\mathcal{Z}^{-1}}{|\vec{x}-\vec{y}|^{\frac{2 \pi}{\beta^{2}}}} \sum_{n=0}^{\infty} C_{n}(|\vec{x}-\vec{y}|)|\vec{x}-\vec{y}|\left(2-\frac{\beta^{2}}{4 \pi}\right) 2 n .
$$

In this expression the coefficients $C_{n}$ are given by the summand in (15) after the $|\vec{x}-\vec{y}|$-factors have been removed. In view of the restriction on the integration region imposed by (16), we see that these coefficients depend, in general, on $|\vec{x}-\vec{y}|$. However, in the large distance regime, where $|\vec{x}-\vec{y}|>>r$, we infer from the right part of (16) that the integration region appearing in the expression of $C_{n}$ is restricted by constant bounds and, therefore, do not depend on $|\vec{x}-\vec{y}|$. Hence, we conclude that the coefficients $C_{n}$ are constant in this limit. This fact is confirmed from an independent point of view in Appendix B.

From Eq. (17) we see that for $\beta^{2}=8 \pi$ we have the following exact expression, valid for the XY-model and the $\mathrm{CG}$ of extensive particles,

$$
\left\langle\mu(\vec{x}) \mu^{\dagger}(\vec{y})\right\rangle=\frac{K(|\vec{x}-\vec{y}|)}{|\vec{x}-\vec{y}|^{\frac{1}{4}}},
$$

where $K(|\vec{x}-\vec{y}|)=\mathcal{Z}^{-1} \sum_{n=0}^{\infty} C_{n}(|\vec{x}-\vec{y}|)$, at $\beta^{2}=8 \pi$.

As we shall prove in Appendix B, $K(|\vec{x}-\vec{y}|)$ is a real function with an upper bound equal to one, which is saturated in the large distance regime, namely, $K(|\vec{x}-\vec{y}|) \stackrel{|\vec{x}-\vec{y}|>>r}{\longrightarrow} 1$. This immediately allows us to write

$$
\left\langle\mu(\vec{x}) \mu^{\dagger}(\vec{y})\right\rangle^{|\vec{x}-\vec{y}|>>>r} \frac{1}{|\vec{x}-\vec{y}|^{\frac{1}{4}}} .
$$

Eq. (19) is the well-known result of Kosterlitz and Thouless for the XY-spin correlation function at the critical temperature $T_{K T}=\pi J$ (remember the relation between the XY-model temperature $T$ and $\beta$, namely, $T=\frac{8 \pi^{2} J}{\beta^{2}}$ ) [2]. Notice that for determining the critical exponent of the static and uniform magnetic susceptibility, only the large distance behavior of the spin correlator is needed. Expression (19) provides an exact result for this. 
We remark, at this point, that only the leading term in the large distance behavior of the spin correlator has been obtained when we took the $|\vec{x}-\vec{y}|>>r$ limit. In order to get the subleading logarithmic correction obtained previously 2 15, namely

$$
\left\langle\mu(\vec{x}) \mu^{\dagger}(\vec{y})\right\rangle \stackrel{|\vec{x}-\vec{y}|>>r}{\longrightarrow} \frac{C(\ln |\vec{x}-\vec{y}|)^{\frac{1}{8}}}{|\vec{x}-\vec{y}|^{\frac{1}{4}}},
$$

we should consider the next term in the $\frac{r}{|\vec{x}-\vec{y}|}$ expansion. According to the bound and asymptotic value obtained for the function $K(x)$ in Appendix $\mathrm{B}$, however, we conclude that the constant $C$ in (20) must be $C=(\ln 2 R)^{-\frac{1}{8}}$ such that

$$
K(|\vec{x}-\vec{y}|) \stackrel{|\vec{x}-\vec{y}|>>r}{\sim} \frac{(\ln |\vec{x}-\vec{y}|)^{\frac{1}{8}}}{(\ln 2 R)^{\frac{1}{8}}} \stackrel{|\vec{x}-\vec{y}|>>r}{\longrightarrow} 1 .
$$

Finally, observe that the exponent $1 / 4$ comes from the free soliton correlator, which is the prefactor in (15) and (17). This expresses the well-known fact that the $\cos \beta \phi$ interaction becomes irrelevant at $\beta^{2}=8 \pi$. Equivalently, in XYmodel language, we would say that the whole contribution to the asymptotic behavior of the correlators comes from the spin-wave term. For $\beta^{2}>8 \pi$, we still have the large distance behavior of the correlators determined by the free theory (spin-wave term). This fact can be inferred directly from (17), even though some logarithmic corrections could be produced by summing in $n$. For $\beta^{2} \geq$ $8 \pi$, a careful analysis of the ultraviolet divergences is required [22] in the local case, where any short distance regulators must be removed at some point. This, however, has no effect on our result (17), where short distance singularities are absent due to the presence of natural regulators.

\section{THE VORTEX CORRELATION FUNCTION}

The large distance behavior of the two-point correlation function of the vortex creation operators in the XY-model can also be obtained exactly within our approach, at the KT point. Here, again, the use of the correspondence with the $\mathrm{SG}$ theory is quite useful. Indeed, from (8) it follows that, in SG language, this operator is given by

$$
\sigma(x, \tau)=\exp \left\{i \frac{\beta}{2} \phi(x, \tau)\right\} .
$$

We can obtain a CG representation for $\left\langle\sigma \sigma^{\dagger}\right\rangle$ analogous to (15). The only differences are the prefactor exponent, which is now $\beta^{2} / 8 \pi$ and the last term, in which $\xi_{i}$ is replaced by $\eta_{i}$ and $i$ by $\beta^{2} / 4 \pi$. Following the same procedure as for the soliton correlation function we obtain, at $\beta^{2}=8 \pi$,

$$
\left\langle\sigma(\vec{x}) \sigma^{\dagger}(\vec{y})\right\rangle=\frac{K^{\prime}(|\vec{x}-\vec{y}|)}{|\vec{x}-\vec{y}|}
$$

where the function $K^{\prime}(|\vec{x}-\vec{y}|)$ can be obtained from $K(|\vec{x}-\vec{y}|)$ through the same replacements described above. In Appendix B we show that $K^{\prime}(|\vec{x}-\vec{y}|)$ presents the same bound and asymptotic limit as $K(|\vec{x}-\vec{y}|)$. 
Thus we have

$$
\left\langle\sigma(\vec{x}) \sigma^{\dagger}(\vec{y})\right\rangle \stackrel{|\vec{x}-\vec{y}|>>r}{\longrightarrow} \frac{1}{|\vec{x}-\vec{y}|}
$$

\section{CONCLUDING REMARKS}

An interesting extension of this work, which we are now considering, is the obtainment of the soliton or XY-vortex four-point correlation functions at the KT point, using the same methodology. It would also be interesting to compare the large distance behavior of our series (15) with the exact solution of the associated free massive fermion theory at the Luther-Emery point $\beta^{2}=4 \pi[19$ 23. This would allow us to determine the coefficients $C_{n}$ at this point. Another interesting issue to be explored would be the large distance behavior of the soliton and vortex correlators in the plasma phase $\left(\beta^{2}<8 \pi\right)$, where an exponential decay should be recovered. Finally, an important case that deserves further investigation is the local SG theory or CG of point particles for $\beta^{2} \geq 8 \pi$, where a careful treatment of the short distance singularities must be performed. 


\section{APPENDIX A}

Here we give details about the bipolar coordinates, which we use for computing the soliton correlation function (14). Given the position vector $\vec{r}$ in the plane and two points (poles) at $\vec{x}$ and $\vec{y}$, we define the coordinates $(\xi, \eta)$ as 24

$$
\begin{gathered}
\xi=\arg (\vec{r}-\vec{y})-\arg (\vec{r}-\vec{x}) \\
\eta=\ln \frac{|\vec{r}-\vec{x}|}{|\vec{r}-\vec{y}|},
\end{gathered}
$$

with $0 \leq \xi \leq 2 \pi$ and $-\infty<\eta<\infty$. In terms of these coordinates, the position vector is given by

$$
\vec{r}=\frac{|\vec{x}-\vec{y}|}{2[\cosh \eta-\cos \xi]}(\sinh \eta, \sin \xi)
$$

and the volume element reads

$$
d^{2} z=\frac{|\vec{x}-\vec{y}|^{2}}{4[\cosh \eta-\cos \xi]^{2}} d \xi d \eta
$$

\section{APPENDIX B}

From (15), (17) and (18) we obtain

$$
\begin{aligned}
& K(|\vec{x}-\vec{y}|)=\mathcal{Z}^{-1} \sum_{n=0}^{\infty} \frac{\alpha^{2 n}}{(n !)^{2}} \int_{V(r)} \prod_{i=1}^{n} d^{2} x_{i} \prod_{i=1}^{n} d^{2} y_{i} \frac{\prod_{i<j}^{n} \frac{\left[x_{i}, x_{j}\right]}{|\vec{x}-\vec{y}|^{4}} \prod_{i<j}^{n} \frac{\left[y_{i}, y_{j}\right]}{|\vec{x}-\vec{y}|^{4}}}{|\vec{x}-\vec{y}|^{4 n} \prod_{i, j}^{n} \frac{\left[x_{i}, y_{j}\right]}{|\vec{x}-\vec{y}|^{4}}} \\
& \times \exp \left\{i \sum_{i=1}^{n}\left[\arg \left(\vec{x}_{i}-\vec{y}\right)-\arg \left(\vec{x}_{i}-\vec{x}\right)\right]-i \sum_{i=1}^{n}\left[\arg \left(\vec{y}_{i}-\vec{y}\right)-\arg \left(\vec{y}_{i}-\vec{x}\right)\right]\right\}
\end{aligned}
$$

In this expression, we went back to usual coordinates and associated $\vec{x}_{i}$ and $\vec{y}_{i}$ with the positive and negative charges, respectively. We also introduced the symbols

$$
\left[x_{i}, y_{j}\right] \equiv\left|\vec{x}_{i}-\vec{y}_{j}\right|^{4}>r^{4} .
$$

Let us first prove that $K$ is real. This can be easily done by taking the complex conjugate of (B.1) and subsequently performing the change of variables $x_{i} \leftrightarrow y_{i}$.

Considering now the contribution of the phase factors to the integrals in B.1. we can establish the following bound:

$K(|\vec{x}-\vec{y}|) \leq \mathcal{Z}^{-1} \sum_{n=0}^{\infty} \frac{\alpha^{2 n}}{(n !)^{2}} \int_{V(r)} \prod_{i=1}^{n} d^{2} x_{i} \prod_{i=1}^{n} d^{2} y_{i} \frac{\prod_{i<j}^{n}\left[x_{i}, x_{j}\right] \prod_{i<j}^{n}\left[y_{i}, y_{j}\right]}{\prod_{i, j}^{n}\left[x_{i}, y_{j}\right]}$

Observe that the $|\vec{x}-\vec{y}|$-terms in (B.1) have completely canceled out. Notice also that the expression given by the sum in $(\mathrm{B} .3)$ is nothing but $\mathcal{Z}$ and therefore we immediately infer that $K(|\vec{x}-\vec{y}|) \leq 1$, which is the announced bound for the function in (18). 
We now consider the asymptotic behavior of $K$, for $|\vec{x}-\vec{y}|>>r$. The phases in (B.1) cancel out in the leading order in this limit and we immediately see that the above bound is saturated in the leading asymptotic regime, namely $K(|\vec{x}-\vec{y}|) \stackrel{|\vec{x}-\vec{y}|>>r}{\longrightarrow} 1$.

In order to complete our analysis, let us establish now an upper bound for $\mathcal{Z}$ itself. Using the expression for $\mathcal{Z}$ given by the sum in B.3. we can infer the following bound for $\mathcal{Z}$, by making the replacement $\left[x_{i}, y_{j}\right] \leftrightarrow r^{4}$ in the $n i=j$ terms in the denominator:

$$
\mathcal{Z} \leq \sum_{n=0}^{\infty} \frac{\left(\frac{\alpha}{r^{2}}\right)^{2 n}}{(n !)^{2}} \int_{V(r)} \prod_{i=1}^{n} d^{2} x_{i} \prod_{i=1}^{n} d^{2} y_{i} f_{n}\left(x_{1}, \ldots, x_{n} ; y_{1}, \ldots, y_{n}\right)
$$

where

$$
f_{n}\left(x_{1}, \ldots, x_{n} ; y_{1}, \ldots, y_{n}\right)=\frac{\prod_{i<j}^{n}\left[x_{i}, x_{j}\right] \prod_{i<j}^{n}\left[y_{i}, y_{j}\right]}{\prod_{i \neq j}^{n}\left[x_{i}, y_{j}\right]} .
$$

Using the Mean-Value Theorem, we may write the $n$-th integral in B.4 as

$$
I_{n}=V^{2 n} \overline{f_{n}}
$$

where $\overline{f_{n}}$ is the average value of $f_{n}$ and $V$ is the "volume" $\left(\simeq \pi R^{2}\right)$ of the system. Observe that $f_{1}=1$, whereas $f_{2}$ is given by

$$
f_{2}=\frac{\left[x_{1}, x_{2}\right]\left[y_{1}, y_{2}\right]}{\left[x_{1}, y_{2}\right]\left[x_{2}, y_{1}\right]}
$$

and

$$
f_{3}=f_{2}\left(x_{1}, x_{2} ; y_{1}, y_{2}\right) f_{2}\left(x_{1}, x_{3} ; y_{1}, y_{3}\right) f_{2}\left(x_{2}, x_{3} ; y_{2}, y_{3}\right)
$$

The generalization for higher values of $n$ is straightforward and we have in general $f_{n}=\left(f_{2}\right)^{\frac{n^{2}-n}{2}}$.

Consider now $I_{2}$. Performing the changes of variables $x_{1} \rightarrow y_{1}$ and $y_{1} \rightarrow x_{1}$, we readily conclude that

$$
\overline{f_{2}}=\overline{f_{2}^{-1}}
$$

This implies that $\overline{f_{2}} \leq 1$ and also that $\overline{\left(f_{2}\right)^{N}} \leq 1$.

Since, as we have just seen, $f_{n}$ is in the form $\left(f_{2}\right)^{N}$ we conclude that $\overline{f_{n}} \leq 1$ and, consequently, $I_{n} \leq V^{2 n}$. Inserting this bound in (B.4), we obtain (notice that $\mathcal{Z}$ is positive)

$$
0<\mathcal{Z} \leq \sum_{n=0}^{\infty} \frac{\left(\frac{\alpha \pi R^{2}}{r^{2}}\right)^{2 n}}{(n !)^{2}}=\mathrm{I}_{0}\left(\frac{2 \pi \alpha R^{2}}{r^{2}}\right)
$$

where $\mathrm{I}_{0}(x)$ is a modified Bessel function of the first kind and we have set $V=$ $\pi R^{2}$, since $R>r$. From this we see that $\mathcal{Z}$ is finite for the $\mathrm{XY}$-model and 
the CG of extensive particles (where there is a natural short distance cutoff $r$ ) whenever the volume is finite .

Now let us consider $K^{\prime}(|\vec{x}-\vec{y}|)$. From the explanations given after (22), we conclude that

$$
\begin{array}{r}
K^{\prime}(|\vec{x}-\vec{y}|)=\mathcal{Z}^{-1} \sum_{n=0}^{\infty} \frac{\alpha^{2 n}}{(n !)^{2}} \int_{V(r)} \prod_{i=1}^{n} d^{2} x_{i} \prod_{i=1}^{n} d^{2} y_{i} \frac{\prod_{i<j}^{n} \frac{\left[x_{i}, x_{j}\right]}{|\vec{x}-\vec{y}|^{4}} \prod_{i<j}^{n} \frac{\left[y_{i}, y_{j}\right]}{|\vec{x}-\vec{y}|^{4}}}{|\vec{x}-\vec{y}|^{4 n} \prod_{i, j}^{n} \frac{\left[x_{i}, y_{j}\right]}{|\vec{x}-\vec{y}|^{4}}} \\
\frac{\prod_{i}^{n}\left[x_{i}, x\right]^{1 / 2} \prod_{i}^{n}\left[y_{i}, y\right]^{1 / 2}}{\prod_{i}^{n}\left[x_{i}, y\right]^{1 / 2} \prod_{i}^{n}\left[y_{i}, x\right]^{1 / 2}}(\text { B.11) }
\end{array}
$$

Observe that the last factor in the previous expression tends to one in the limit $|\vec{x}-\vec{y}|>>r$. Hence, we immediately conclude, after the $|\vec{x}-\vec{y}|$-terms are canceled out, that $K^{\prime}(|\vec{x}-\vec{y}|) \stackrel{|\vec{x}-\vec{y}|>>r}{\longrightarrow} 1$, thus establishing (24).

\section{ACKNOWLEDGMENTS}

This work has been supported in part by CNPq, FAPERJ and PRONEX66.2002/1998-9. LM was supported by CNPq and ECM was partially supported by CNPq.

\section{References}

[1] S. Samuel, Phys. Rev. D 18:1916 (1978).

[2] J. M. Kosterlitz, J. Phys. C: Solid State Phys. 7:1046 (1974).

[3] A. B. Zamolodchikov and Al. B. Zamolodchikov, Ann. Phys. (NY) 120:253 (1979); V. E. Korepin, Commun. Math. Phys. 76:165 (1980).

[4] A. Luther and V. J. Emery, Phys. Rev. Lett. 33:589 (1974).

[5] C. Destri and H. J. de Vega, Nucl. Phys. B 438:413 (1995).

[6] S. Lukyanov and A. Zamolodchikov, Nucl. Phys. B 493:571 (1997).

[7] S. Lukyanov and A. Zamolodchikov, Nucl. Phys. B 607:437 (2001).

[8] L. Samaj and B. Jancovici, J. Stat. Phys. 106:323 (2002).

[9] L. Samaj, J. Phys. A: Math. Gen. 36:5913 (2003).

[10] L. Samaj and I. Travenec, J. Stat. Phys. 101:713 (2000).

[11] P. Kalinay and L. Samaj, J. Stat. Phys. 106:857 (2002).

[12] A. Alastuey and F. Cornu, J. Stat. Phys. 66:165 (1992).

[13] J. M. Kosterlitz and D. J. Thouless, J. Phys. C: Solid State Phys. 6:1181 (1973).

[14] J. V. José et al., Phys. Rev. B 16:1217 (1977).

[15] D. J. Amit et al., J. Phys. A: Math. Gen. 13:585 (1980).

[16] T. Giamarchi and H. J. Schulz, Phys. Rev. B 39:4620 (1989).

[17] P. Minnhagen, Rev. Mod. Phys. 59:1001 (1987).

[18] P. J. Forrester et al., J. Stat. Phys. 84:359 (1996).

[19] S. Mandelstam, Phys. Rev. D 11:3026 (1975).

[20] A. M. Tsvelik, Quantum Field Theory in Condensed Matter Physics, Cambridge, 1995.

[21] E. C. Marino and J. A. Swieca, Nucl. Phys. B 170[FS1]:175 (1980).

[22] A. Lima-Santos and E. C. Marino, J. Stat. Phys. 55:157 (1989).

[23] S. Coleman, Phys. Rev. D 11:2088 (1975).

[24] G. Arfken, Mathematical Methods for Physicists, $2^{\text {nd }}$ edition, Academic Press, Inc., New York, 1970. 